\title{
Entscheide der Paritätischen Interpretationskommission
}

TARMED Suisse

\author{
Nummer 06026: Bestrahlungspläne \\ Tarifposition: 32.0240 und 32.0260 \\ Gültig ab: 14. Dezember 2006
}

\section{Interpretation}

Ein Elektronenboost ist in der Regel eine neue Bestrahlungsphase (meist nur die letzten paar Tage einer mehrwöchigen Behandlung) und kann und soll somit separat geplant werden. Es handelt sich auch nicht um das gleiche, sondern um ein neu definiertes, klinisch oder radiologisch festzustellendes Bestrahlungsvolumen. Zudem richtet es sich zum Beispiel nach der noch verbliebenen Grösse eines Tumorrestes. Es kann auch vorkommen, dass gleichzeitig die Bestrahlung mehrerer Volumina begonnen wird, z.B. eine 3-D geplante Bestrahlung eines grossen Beckentumors und eine ohne Computer geplante Bestrahlung einer Rippenmetastase mit einem Elektronenfeld. Auch in diesem Fall ist die Abrechnung beider Positionen gerechtfertigt. In den beiden beschriebenen Fällen handelt es sich nicht um Kumulationen der beiden betreffenden Positionen.

Die PIK ist aber einverstanden mit einem Kumulationsverbot von Bestrahlungsplänen für das gleiche Volumen in der gleichen Bestrah- lungsphase. Wenn ein Elektronenfeld Teil eines Photonenplanes ist, kann es gleichzeitig mit diesem Plan am Planungscomputer berechnet werden.

Die Interpretation wird wie folgt ergänzt:

\subsection{0}

Menge: 1mal pro Bestrahlungsphase und Volumen, nicht kumulierbar mit 32.0240.

\subsection{0}

Menge: 1mal pro Bestrahlungsphase und Volumen, nicht kumulierbar mit 32.0260.

\section{Nummer 06028: 02.0260, Leistung in Abwesenheit des Patienten durch delegierten Psychologen/Psycho- therapeuten, pro 5 Min.}

Tarifposition: 02.0260

Gültig ab: 14. Dezember 2006

\section{Interpretation}

Berichte gemäss Art. 3, KLV sind mit den Pos. 00.2285 (nichtformalisierter Bericht, 11 bis 35 Zeilen Text) und 00.2295 (+ nichtformalisierter Bericht, jede weiteren 35 Zeilen Text) abzurechnen. 